\title{
Annular Arrays for Novel Measurement Methods
}

\author{
Elfgard Kühnicke, Mario Wolf, Sebastian Kümmritz, Michael Lenz \\ Solid-State Electronics Laboratory; Dresden University of Technology, Mommsenstr. 10, \\ 01069 Dresden, Germany \\ elfgard.kuehnicke@tu-dresden.de
}

\begin{abstract}
:
The paper shows how the precise knowledge of the sound field of an ultrasonic annular array can contribute to the development of novel measuring techniques. Emphasis is placed on a) a noninvasive method for sound velocity measurements in fluids using the echo signals from scattering particles, b) a non-invasive method for the combined determination of thickness and sound velocity in layered structures by using a novel focusing technique, and c) a non-scanning curvature measurement method exploiting the wave front curvature of a reflected wave. To demonstrate the methods, the principles as well as results from simulations and measurements are discussed.
\end{abstract}

Key words: annular array, sound velocity, thickness, curvature, scattering particles

\section{Introduction}

In ultrasonic measurements, the time of flight to an interface of an object is often the only evaluated information. This paper demonstrates how additional information of the reflected sound field is gained by using segmented annular arrays. New measurement techniques have been developed which are characterized by a close combination of sound field simulations and measurements. They use synthetic or electronic focusing and analyze the phases and amplitudes on all transducer elements to determine the object curvature radius or sound velocity and distance simultaneously.

These approaches require the precise knowledge of the sound field in dependence on the array parameters and the set-up. Therefore, sound field simulations are necessary.

A half-analytical method has been developed to optimize transducers and to calculate the sound field in complex structures and layered media. It is based on time-harmonic GREEN's functions in a steepest descent approximation for a twodimensional geometry. The source area is discretized and uniformly covered with point sources. The sound field that is irradiated from a finitely extended source is the superposition of the field of all point sources. The sound field in a layered medium can be calculated approximately by a decomposition of the medium into different layers and a separate calculation of wave propagation in each layer [1]. This method is applied for each element to simulate the field generated in the medium or at the different interfaces, including the reflections back to the transducer. The applied focusing techniques can be realized by phase shifting and superposing the different fields.

Different annular arrays are used (Fig. 1). They have six active ring elements and, depending on the task, a curved interface for a small focus extension or additional segments for non-axissymmetric problems or adjustment (Fig. 1 right).
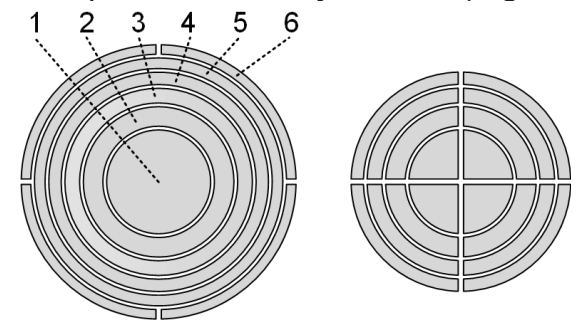

Fig. 1. Geometry of the annular arrays.

\section{Scattering particle method for locally re- solved measurement of sound velocity}

The monitoring of sound velocity is of great interest in chemical processes, because sound velocity is a measure for concentration, mass density and elastic parameters. Other possible applications of locally resolved sound velocity measurements are tissue characterization and 
noninvasive temperature monitoring during hyperthermia for cancer therapy.

Conventional pulse-echo methods measure the time of flight to determine only one quantity: the sound velocity or the distance of a reflector, where the other quantity has to be known. To measure a local distribution of sound velocity, usually further reflectors or transducers at known positions are required. These additional mechanical parts cause a high instrumentation effort. If the examined medium is not all-round accessible, transducers/reflectors have to be inserted, which can affect the examined process. Therefore, a non-invasive method without the need for fixed reflectors is most desirable to measure sound velocity profiles.

This paper presents a novel approach for simultaneous measurement of sound velocities and distances in fluids containing moving scattering particles. Because the sound field of an ultrasonic transducer depends on both the transducer parameters and the sound velocity of the propagation medium, the focus position is used as a second measuring quantity instead of using the propagation time alone. The focus position is determined by evaluating the echoes of the scatterers. The echo of a single particle becomes strongest if it is located in the focal zone of the sound field. Because particles are at motion in a fluid, it is possible to assume a uniform distribution of particles in time average. So the averaged amplitude of echo signals becomes maximal for the time of flight to the focus and back (Fig. 2).

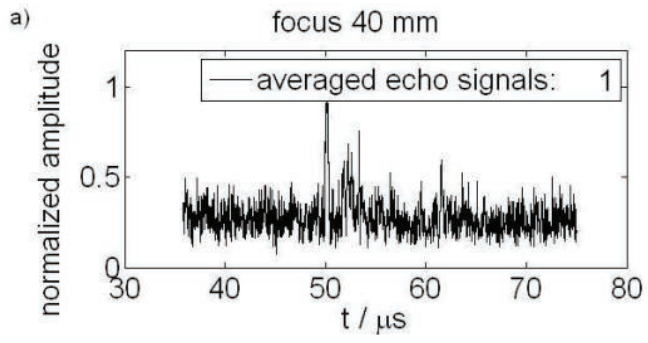

b)

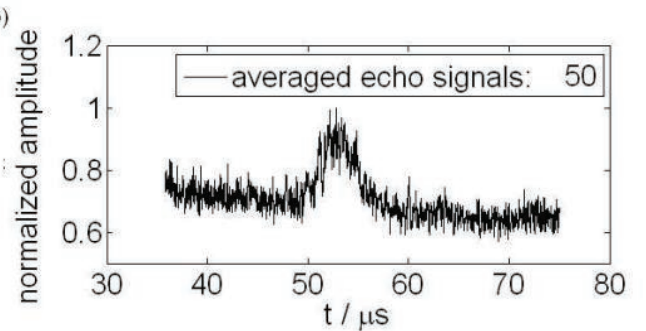

Fig. 2. Normalized amplitude of averaged echo signals of an annular array at focussing Fok $=40 \mathrm{~mm}$ in water of $30^{\circ} \mathrm{C}(\mathrm{c}=1510 \mathrm{~m} / \mathrm{s})$ for different numbers of averages (Maximum at $t=52 \mu \mathrm{s}$.)
Papers [2] and [3] give proof of concept by measurements in fluids covering a wide range of sound velocities with a strongly focusing transducer equipped with a lens. An uncertainty in velocity determination of less than $0.1 \%$ was achieved in a detailed measurement using water at different temperatures.

To achieve a local resolution of the sound velocity measurement along the axis without additional mechanical effort, an ultrasonic array is required. Due to the available electronic instrumentation, first measurements were done by emission with one element and synthetic focusing of the received signals, which means that signals are time shifted and superposed [4]. The development of a new pulser allowed driving all elements together, whereby each pulse can be delayed with a definite time lag. For a second focusing, the received signals are superposed and thus focused synthetically. This reduces the statistical uncertainty, because it minimizes the focus extension and contributes to a larger signal to noise ratio.

The control mode Fok corresponds to a set of delay times. The delay times are chosen in such a way that the focus of the transducer is located at the distance $z=$ Fok in the calibration medium water at room temperature. This set of delay times is used for all measurements, independently from the real sound velocity.

Because the focus position depends on the sound velocity of the medium, sound field simulations are required to predict the focus position as a function of the sound velocity of the medium, where the parameters of the array need to be considered, as well. Fig. 3 shows calculated sound fields of the used curved annular array for two different control modes and for two different sound velocities of the propagation medium.

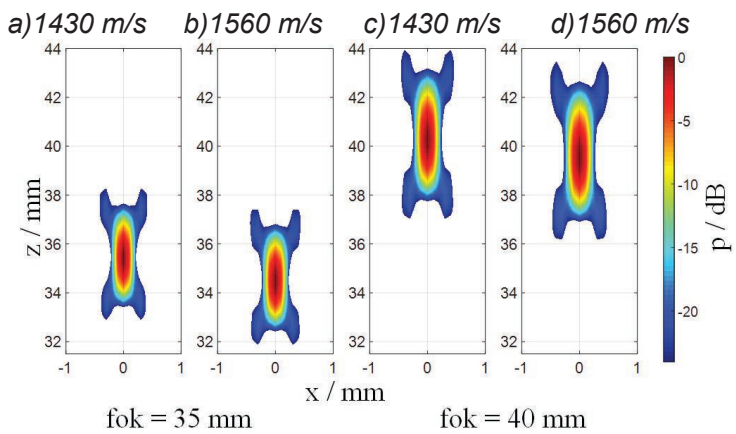

Fig. 3. Calculated sound fields (focusing in send and receive mode) in dependence of the sound velocity in the propagation medium for a curved $9 \mathrm{MHz}$ annular array at different control modes Fok. 
Fig. 3 confirms that the focus position depends on the velocity of the propagation medium (compare Fig. 3a with $3 b$, and $3 c$ with $3 d$ ). The position of the sound field maximum can be varied by means of control mode (compare Fig. $3 a$ with $3 c$, and Fig. $3 b$ with $3 d$ ).

To create calibration curves, the sound fields are simulated for different sound velocities (and different control modes). The positions of the sound field maxima are then converted into the corresponding times of flight. Plotting the times of flight as a function of sound velocity for several control modes Fok leads to the series of calibration curves depicted in Fig. 4. Using the measured time of flight in an unknown fluid and considering the used control mode Fok then enables us to read off the sound velocity of the medium using Fig. 4.

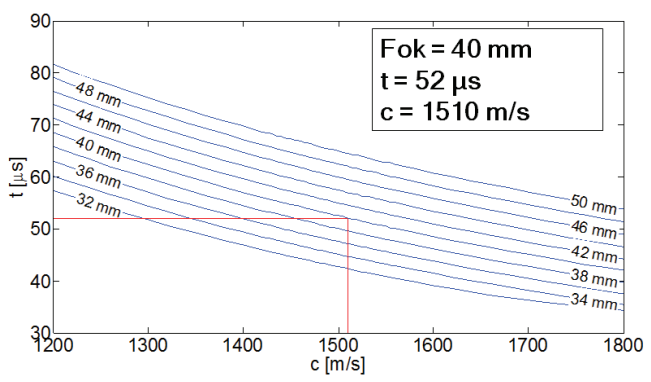

Fig. 4. Calibration curve as a function of sound velocity for different control modes.

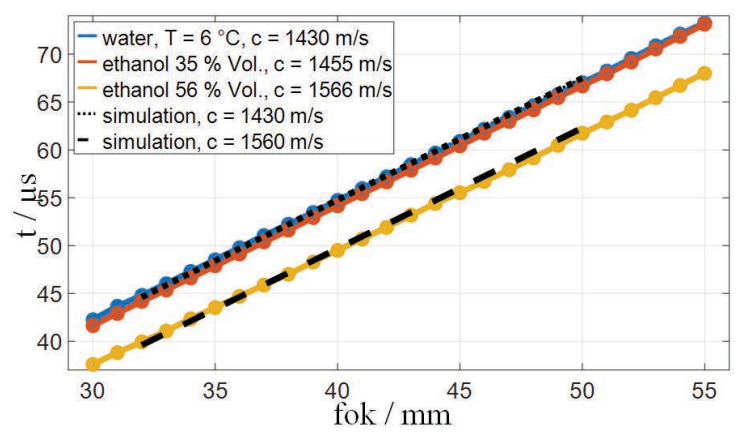

Fig. 5. Time of flight to the sound field maximum as a function of sound velocity and the control mode Fok

To compare measurements and simulations, echoes of scattering particles and so sound velocities and focus positions were measured in media with constant temperature (Fig. 5). It is striking that the measured curves fit very well to the simulations.

The method has been qualified for media with constant sound velocity, and for these measurements, an accuracy of more than $99.8 \%$ was reached [5]. It had been shown that the measurement uncertainty for constant sound velocities can be reduced to $2.5 \mathrm{~m} / \mathrm{s}$, with is less than $2 \%$, in water (see Tab. 1 ).
Tab. 1: Reachable uncertainty.

\begin{tabular}{|l|l|l|}
\hline $\begin{array}{l}\text { number of aver- } \\
\text { aged signals }\end{array}$ & $\begin{array}{l}\text { standard deviation } \\
\pm 3 \sigma \text { of ToF }\end{array}$ & $\begin{array}{l}\text { absolute error of } \\
\text { sound velocity }\end{array}$ \\
\hline 50 & $500 \mathrm{~ns}$ & $13 \mathrm{~m} / \mathrm{s}$ \\
\hline 200 & $250 \mathrm{~ns}$ & $6 \mathrm{~m} / \mathrm{s}$ \\
\hline 1000 & $150 \mathrm{~ns}$ & $4 \mathrm{~m} / \mathrm{s}$ \\
\hline 5000 & $100 \mathrm{~ns}$ & $2.5 \mathrm{~m} / \mathrm{s}$ \\
\hline
\end{tabular}

The current work deals with the generating of calibration curves for media with continuously changing properties. The continuous change of material properties is taken into account by a modified wave equation. The potential of a point source has been calculated in the HANKEL transformed domain. The inverse transform is realized and allows calculating GREEN's functions for media with continuously changing properties for a change of material properties in axial direction of the array [6]. A change of these properties in other directions would cause a lateral deviation of the focus position and has to be examined in further works. Further advances of the method are planned for measuring sound velocity profiles in media with stationary particles as e. g. medical tissues.

\section{Simultaneous Determination of Thickness and Sound Velocity}

For imaging in NDT or in medical diagnostics, the value of sound velocity is assumed a priori. Interfaces of hidden objects (defects, surfaces of organs, vessels) are imaged by the measured time of flight (ToF). The supposed locations and the extensions of objects are incorrect if the real speed of sound differs from the assumption.

The thickness of a specimen is measured e. $\mathrm{g}$. mechanically for material characterization, and the sound velocity is determined by ToFmeasurements. If there is a multi-layered structure, the mechanical determination of the thickness of the different layers is impossible nondestructively. It is necessary to determine both quantities simultaneously to get information about the thicknesses and the materials of the different layers.

To satisfy the demands of NDT, novel approaches evaluate the sound field, which depends on the propagation medium. Gust [7] determines sound velocity and thickness of plates with a scanning acoustic microscope by moving a probe in water vertically to the surface. The focus position is used as a second measuring quantity additional to the propagation time. The echo of the surface and of the back wall is maximal if the focus is located on it 
(compare signals for position 2 and 3, Fig. 6). The displacement between the transducer positions, where the echo of the first and the second interface become strongest, is determined. Because the focus position depends on the sound velocity, the necessary displacement differs in dependence on the material of the plates, even for plates of the same thickness. So the displacement and the time of flight between the interfaces can be used to determine sound velocity and thickness simultaneously.

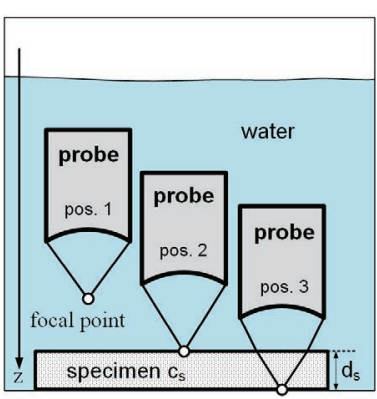

time signals for the different probe positions

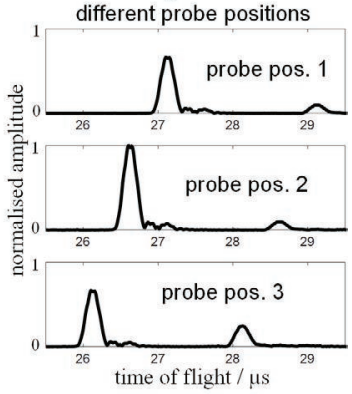

Fig. 6. Principle drawing for the combined measurement of thickness and sound velocity by means of a focusing transducer.

A method is introduced in [8] allowing a simultaneous determination of sound velocity and thickness of up to two layers with an annular array at a fixed position. It works by varying the control mode to move the focus position along the acoustic axis. The amplitudes of the echo signals are evaluated to get additional information besides the time of flight. A measurement set-up with a water delay line between the specimen and a plane annular array with a centre frequency of $6 \mathrm{MHz}$ is used.

The approach is explained by analyzing a first, liquid layer. The focus position is continuously moved along the acoustic axis by changing the control mode. In contrast to former measurements, the control modes are calculated on the basis of different predetermined sound velocities $C_{\text {control, }}$ here. In Fig. 7 (right curve), a control sound velocity of $1000 \mathrm{~m} / \mathrm{s}$ is used to calculate the delay times. The back wall echo signal is determined, and its amplitude is recorded as a function of the control mode. The result is the blue focusing curve on the right side. At $30 \mathrm{~mm}$, a maximum can be seen. This maximum indicates that if the sound velocity of the fluid was $1000 \mathrm{~m} / \mathrm{s}$, the thickness of the first layer would be $30 \mathrm{~mm}$. The process is repeated for different control velocities $\mathrm{c}_{\text {control }}$ (Fig. 7), and the supposed thickness is recorded as a function of control sound velocity (Fig. 8, blue line).

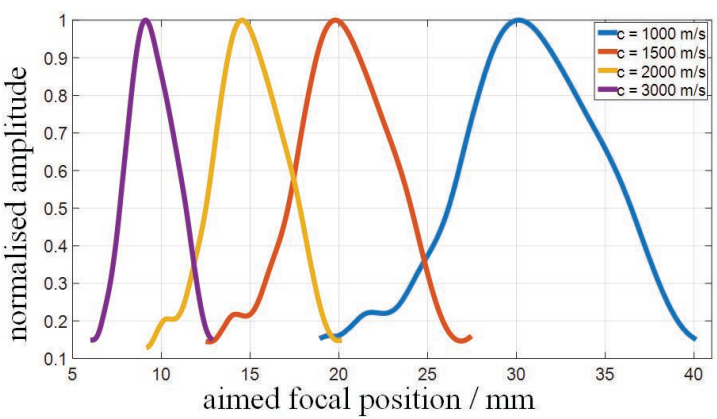

Fig. 7. Focusing curves for different control sound velocities $\mathrm{C}_{\text {control. }}$

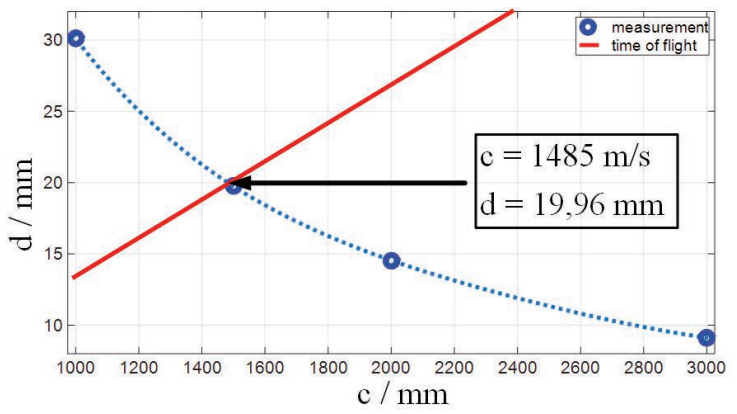

Fig. 8. Determination of sound velocity by intersection of two curves; Red curve: reflector distance as a function of sound velocity and measured ToF. Blue curve: Reflector distance as a function of the control sound velocity control; Blue points: Four pairs of sound velocity and corresponding distance are determined by focusing curves of Fig. 7.

The ToF between probe and surface gives additional information (red line). The correct sound velocity and thickness of the specimen is the intersection of the two curves.

After the determination of thickness and velocity of the first layer, the approach is repeated for the second layer. Refraction effects have to be taken into account to correctly focus into the second medium. The delay times are determined by means of Fermat's principle. The thickness and sound velocity of the first layer have to be known for this.

Measurements were done for a two-layered structure with the first layer of water and the second layer of steel or aluminum with a thickness $d=6 \mathrm{~mm}, 8 \mathrm{~mm}, 10 \mathrm{~mm}, 12 \mathrm{~mm}$ and $14 \mathrm{~mm}$ [8]. The relative error was less than $1 \%$. For the second layer, a relative error of less than $3 \%$ had been achieved for the current measurement set-up.

The main problem is that this analysis uses only a geometric model neglecting the wave properties. It cannot ensure the same accuracy if the measurement set-up is modified. Alternatively, in [9], a fast and efficient method for a simulta- 
neous determination of sound velocity and thickness of a two-layered structure has been presented. It analyses the different signal parts of an echo reflected from the examined interface. These signal parts correspond to different propagation paths (Fig. 9). The time difference between the signal parts contains the information about thickness and sound velocity of the layer. These time differences are used as an input for an inverse geometric model.

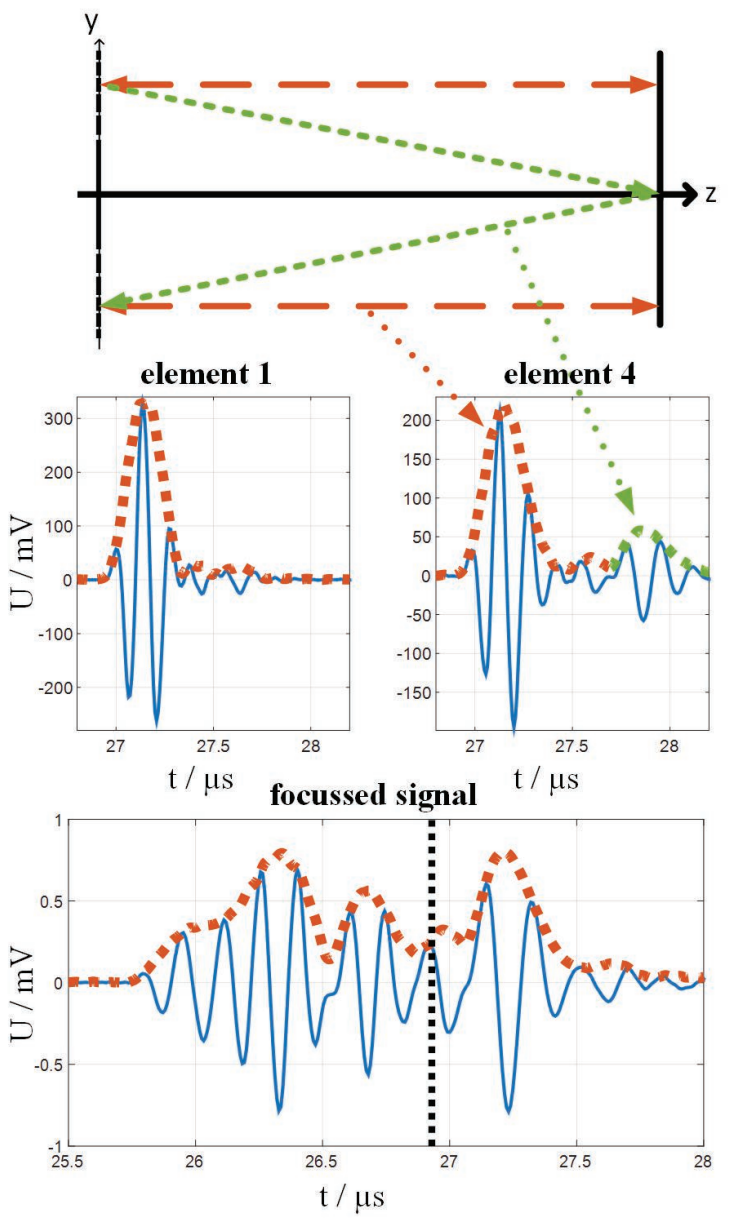

Fig. 9. Signals for different propagation paths.

The feasibility of the method has been proved. For the second layer, an accuracy of $95 \%$ had been reached. Currently, sound field simulations are done to generate a wave-based model of propagation times.

\section{Curvature measurements}

When measuring in pulse-echo mode, the reflected ultrasonic wave does not only contain information about material parameters such as the sound velocities in a layered structure, but also some information about the local inclination angle toward the acoustic axis and the curvature of the reflector.
While the information about the reflector inclination is mainly contained in the angle of reflection of the reflected wave, the information about the reflector curvature is contained in the wave front curvature and the arrival times of the reflected wave on the different elements of an annular, respectively.

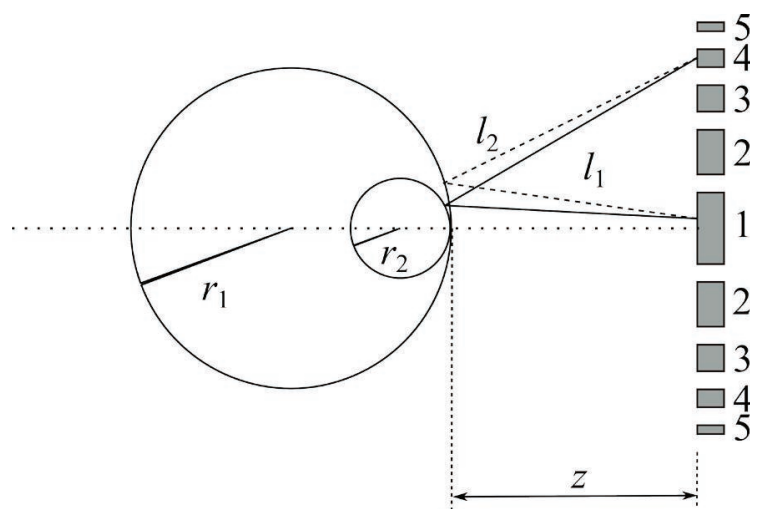

Fig. 10. The time of flight of the ultrasonic wave between the inner and outer transducer elements (numbers 1-5) of an annular array depends on the reflector curvature. The larger the sphere, the shorter is the propagation path from the innermost element to the outer elements.

In Fig. 10 it can be seen that the propagation path lengths between the inner element of an annular array and the outer elements show a dependence on the reflector radius.
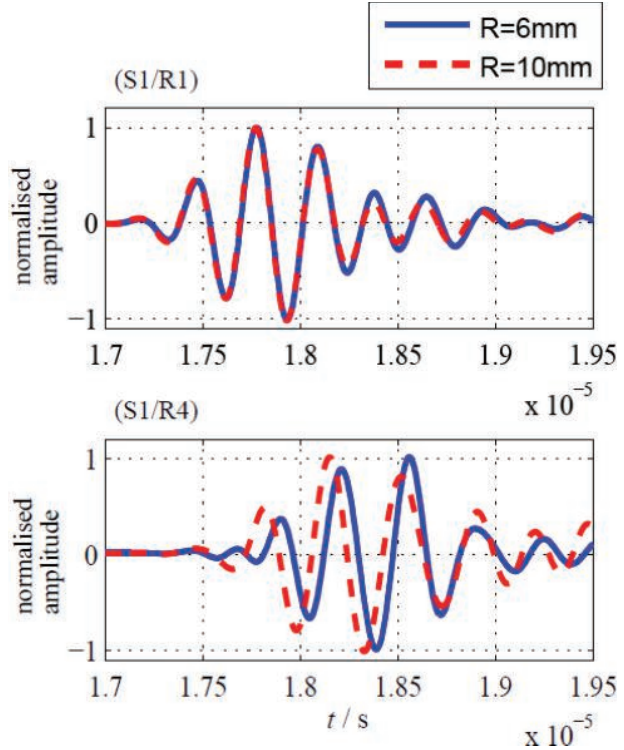

Fig. 11. Measured echo signals for the propagation paths (S1/R1) and (S1/R4) of a plane annular array with five elements for sphere radii of 6 and $10 \mathrm{~mm}$ (S: sending element, $R$ : receiving element). The sphere is located at $z=13 \mathrm{~mm}$, which is the near field length of the single elements of the annular array.

In. Fig. 11, measurements were done to distinguish reflectors and determine the reflector curvature of shperes being kept in a water bath. 
While the time of flight of the wave propagating along the acoustic axis is the same for both reflector radii (Fig. 11, upper figure), the time of flight from the innermost element to the outer element with number four depends on the reflector radius (Fig. 11, lower figure). Under the given conditions, sphere radii could be determined with an uncertainty of about $0.5 \mathrm{~mm}$, where spheres of between $6 \mathrm{~mm}$ and $11 \mathrm{~mm}$ radius were used in the experiments [10].

A further segmentation of the annular array in four $90^{\circ}$ pieces enables the differentiation of the half axes of a rotational ellipsoid. In the following measurements, the $90^{\circ}$-segments on the opposite sides of the transducer were always used together, where the half axes of the reflector were put parallel to the symmetry axes of the transducer (Fig. 12, upper figure).

Instead of measuring time delays of different propagation paths, as in the set-up of Fig. 11, the transducer was focused, here, and the echo signal amplitude was plotted as a function of the focus setting Foc, i. e. the set of delay times focusing at a distance of $z=F O c$ on the acoustic axis. As can be seen in Fig. 12 (lower figure), the different half axes of an elliptical reflector can be distinguished by comparing the maximum positions of the focusing curves.

While the main drawback of the presented nonscanning curvature measurement methods are the necessity for large transducer apertures and the high cross-sensitivity of the method towards local inaccuracies of the surface, the methods might be useful in the future to achieve addi-

\section{References}

[1] E. Kühnicke, Plane arrays - Fundamental investigations for correct steering by means of soun field calculations, Wave Motion 44, 248-261 (2007); DOI:10.1016/j.wavemoti.2006.10.003

[2] M. Lenz, M. Bock, E. Kühnicke, J. Pal, and A. Cramer, Measurement of the sound velocity in fluids using the echo signals from scattering particles, Ultrasonics (2011); DOI: 10.1016/j.ultras.2011.07.003

[3] Lenz M, Kühnicke E, Nichtinvasive Schallgeschwindigkeitsmessung in Fluiden auf Basis von Streupartikelechos, $t m$ - Technisches Messen 79/1, 23-28 (2012); DOI: 10.1524/teme.2012.017

[4] E. Kühnicke, M. Lenz, M. Bock, Non-invasive measurement of sound velocity profiles, IEEE International Ultrasonics Symposium, 2151-2153 (2011).

[5] M. Wolf, E. Kühnicke, M. Lenz., Modeling of sound propagation in media with continuously changing properties towards a locally resolved measurement of sound velocity, IEEE Interna- tional pieces of information in scanning and non-scanning applications in NDT and medical engineering.
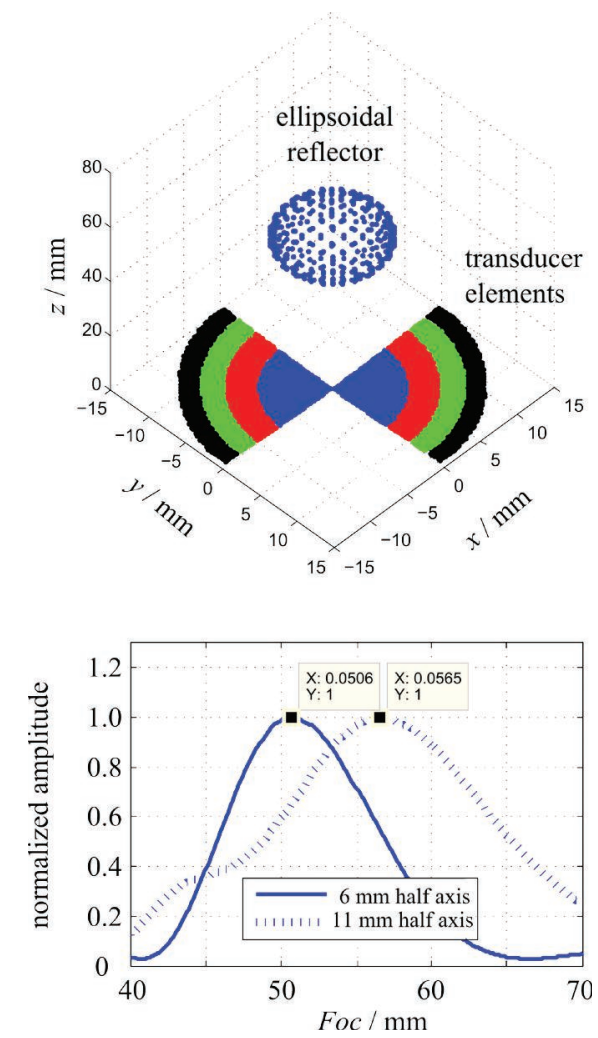

Fig. 12. Focused sound emission with two opposite $90^{\circ}$ transducer segments each being divided in radial direction into four elements (upper figure). The focusing curves allow the determination of the half-axes of the reflector (lower figure).

tional Ultrasonics Symposium (IUS), 1045-1048 (2013)

[6] M. Wolf, E. Kühnicke, Non-invasive and Locally Resolved Measurement of Sound Velocity by Ultrasound, Sensors \& Transducers 184/1, 53-59, (2015)

[7] N. Gust, Improvement of signal analysis for ultrasonic microscopy, Dissertation Techn. Universität Dresden, (2011); ISBN 978-3-942710-22-0

[8] S. Kümmritz, M. Wolf, E. Kühnicke, Simultan Bestimmung von Dicken und Schallgeschwindigkeiten geschichteter Strukturen, tm - Technisches Messen 82/3, (2015)

[9] M. Wolf, S. Kümmritz, E. Kühnicke, Advance signal analysis for the examination of multi- layered structures using annular arrays, Conference Proceedings ECNDT Prag, (2014)

[10] M. Lenz, Neuartige Ultraschallmeßverfahren unter Nutzung von Schallfeldinformationen, Dissertation Technische Universität Dresden, (2013). http://nbn-resolving.de/urn:nbn:de:bsz:14qucosa-135656 